\title{
INVESTIGACIONES
}

\section{Estudiante de docencia en matemáticas y la construcción de la identidad disciplinar*,**}

\author{
Student for mathematics teachers and the construction of the disciplinary identity
}

\author{
Claudio Enrique Opazo Arellano ${ }^{a}$, Francisco Cordero Osorio $^{b}$ \\ ${ }^{a}$ Centro de Investigación y de Estudios Avanzados del Instituto Politécnico Nacional (Cinvestav-IPN), México. \\ copazo@cinvestav.mx \\ ${ }^{b}$ Centro de Investigación y de Estudios Avanzados del Instituto Politécnico Nacional (Cinvestav-IPN), México. \\ fcordero@cinvestav.mx
}

\section{RESUMEN}

En esta investigación ofrecemos evidencia empírica de que la identidad disciplinar es un factor de la formación de los estudiantes de docencia en matemáticas. Mostramos, con la Teoría Socioepistemológica de la Matemática Educativa, cómo ese factor interviene en la resignificación del conocimiento matemático y en la definición de la función del docente de matemáticas. La fuente de análisis se encontró en el escenario escolar-académico del estudiante acompañado del desarrollo de la visión de la enseñanza y la del conocimiento matemático que va construyendo en el hábito permanente de los diseños de actividades escolares. Con métodos etnográficos y entrevistas semiestructuradas se realizaron inmersiones con comunidades de estudiantes que se están formando en la docencia. Como resultado de la investigación se encontró que en el hábito permanente del diseño de actividades escolares está la fuente de sentido que define la identidad disciplinar.

Palabras claves: Formación del profesor, escenario escolar-académico, diseños de actividades escolares.

\begin{abstract}
In this research we offer empirical evidence that disciplinary identity is a factor in the training of students in mathematics teaching. We show, with the Socioepistemological Theory of Mathematics Education, how this factor intervenes in the resignifications of mathematical knowledge and in the definition of the mathematics teacher function. The source of analysis was found in the student's school-academic setting accompanied by the development of the vision of teaching and mathematical knowledge that is building on the permanent habit of school activity designs. Using ethnographic methods and semi-structured interviews, immersions were conducted with communities of students who are being trained in teaching. As a result of the research it was found that in the permanent habit of designing school activities is the source of meaning that defines the disciplinary identity.

Key words: Teacher training, school-academic setting, designing school activities.
\end{abstract}

\footnotetext{
* Esta investigación está financiada por CONACYT con el proyecto, Una categoría de modelación matemática. La pluralidad epistemológica y la transversalidad de saberes: los aprendizajes de los significados de la matemática en las ingenierías y en los diferentes niveles educativos. Clave 0284259.

** Agradecemos la enorme colaboración del Dr. Héctor Alejandro Silva Crocci, profesor-investigador de la Universidad de Santiago de Chile. Su ayuda fue fundamental para conocer la comunidad de estudiantes de docencia de la matemática.
} 


\section{INTRODUCCIÓN}

En la formación inicial docente el único referente de la matemática que tiene el estudiante de docencia es el escolar. Sin embargo, la demanda educativa de la matemática exige diferentes líneas de acción. Por un lado, la diversidad de saberes matemáticos para que sucedan los aprendizajes en situaciones específicas. Por otro, la transversalidad de saberes entre los diferentes niveles educativos. No considerar estas líneas de acción conlleva no confrontar el saber matemático escolar con otros saberes de la matemática. Entonces, ese referente escolar debe ser ampliado al considerar saberes matemáticos de la escuela, del trabajo y de la vida. Quizá la ampliación son los referentes de la matemática que definen el saber de la matemática del docente. Pero vale la pena llamar la atención de que esos saberes ampliados no son componentes de la matemática escolar habitual, más aún son opacados por la epistemología dominante que impone el discurso Matemático Escolar (dME). Romper con esa epistemología requiere forjar una fuente de sentido que permita enfrentar el rompimiento y favorecer el camino a una nueva epistemología (Opazo-Arellano, Cordero y Silva-Crocci, 2018).

Una característica de la epistemología dominante es normar la enseñanza habitual de la matemática escolar a partir de definiciones y conceptos. Por lo que, se privilegian argumentos que se adquieren y reproducen a la luz de la resolución de problemas exógenos del cotidiano del estudiante que se forma en la docencia de la matemática. Estos argumentos se aceptan, no se cuestionan, menos se trastocan. Lo que deriva en adherencia al dME en la formación inicial docente (Cordero y Silva-Crocci, 2012; Cordero, Gómez, Silva-Crocci y Soto, 2015).

La adherencia implica no confrontar la matemática escolar, por lo que el estudiante que aprende para enseñar adopta la epistemología dominante. Una consecuencia es que los usos y significados del conocimiento matemático que emergen en su cotidiano escolar, del trabajo y de la vida no se valorizan en la construcción de su visión de la enseñanza y del conocimiento matemático (Opazo-Arellano, Cordero y Silva-Crocci, 2019). Esta es la problemática de nuestra investigación.

Una pregunta que surge en este contexto es, ¿cómo el estudiante que se está formando para ser docente de matemática puede encarar la adherencia? Con el programa socioepistemológico Sujeto Olvidado y la Transversalidad de Saberes ${ }^{1}$ se ha desarrollado el constructo identidad disciplinar. Es uno de los multifactores que coadyuvan a resignificar ${ }^{2}$ el conocimiento matemático y por ende trastoca la formación inicial docente; pero, también, es uno de los factores que define el nuevo papel del docente, que aquí le llamamos la función del docente (Opazo-Arellano, Cordero y Silva-Crocci, 2018).

La identidad disciplinar trastoca la formación inicial habitual ya que los que aprenden para enseñar legitiman un conocimiento matemático que a priori no está en la enseñanza de la matemática escolar. Además, la identidad compenetrada en la formación hace que el estudiante pase por un proceso de resistencia, cuando pone en uso su visión de la

El objetivo del programa Sujeto Olvidado y la Transversalidad de Saberes (SOLTSA) es revelar los usos del conocimiento matemático y sus resignificaciones en las comunidades de conocimiento de la gente (Cordero, 2016a; Cordero, 2016b; Cordero, 2017). En la siguiente sección del artículo se abordan con mayor profundidad sus alcances.

2 La resignificación expresa la movilidad de los usos y significados del conocimiento matemático en las diferentes situaciones específicas, propias de otros dominios de conocimiento y del cotidiano de la vida (Mendoza-Higuera, Cordero, Solís y Gómez, 2018). 
enseñanza y del conocimiento matemático en diferentes escenarios. Pero también, proyecta la resignificación del conocimiento matemático al crear y mantener diseños de actividades escolares que valorizan la pluralidad epistemológica ${ }^{3}$ y la transversalidad ${ }^{4}$ de saberes en sus experiencias de campo.

Esta tesis de identidad disciplinar está inscrita en la naturaleza del saber matemático en la docencia. La razón es que, por un lado, este saber es la puesta en uso de la matemática del estudiante de docencia a través de dos grandes ejes: la pluralidad epistemológica y la transversalidad de saberes; y, por otro, se confronta con la epistemología dominante que prevalece en la matemática escolar. Para resistir la confrontación son obligadas las acciones de la identidad disciplinar.

La identidad disciplinar abarca una perspectiva que asume que los problemas de enseñanza y aprendizaje de las matemáticas son fenómenos de naturaleza social, los cuales deben ser estudiados a la luz de la Construcción Social del Conocimiento Matemático (CSCM). Es el caso de la adherencia, la exclusión y la opacidad (Cordero et al., 2015). Estos estudios critican a la habitual matemática escolar carente de marcos de referencia para resignificar el conocimiento matemático en situaciones diversas. A priori, en la matemática escolar, no está el rol de la diversidad de saberes matemáticos.

Sin embargo, reconocemos que hay líneas de investigación de gran envergadura que contribuyen a la problemática de la formación del docente de matemáticas en el mundo. Por ejemplo, Godino, Giacomone, Batanero y Font (2017), ofrecen un estudio sobre la necesidad de mejorar la optimización de los recursos que disponen los docentes para la enseñanza de la matemática escolar. En el estudio solo se considera el saber matemático escolar habitual, no se amplía a otros saberes. Sería interesante conocer cómo se afecta la optimización cuando se valorizan los usos del conocimiento matemático del que estudia la docencia de la matemática orientada a la enseñanza media ${ }^{5}$.

Pero también hay investigaciones que enfocan la atención al conocimiento matemático para la enseñanza. Por ejemplo, el Mathematical Knowledge for Teaching, conocido como el MKT (por sus siglas en inglés), propuesta realizada por Ball, Thames y Phelps (2008), cuyo foco de atención está en el análisis del conocimiento del profesor y el diseño de tareas que mejoren su práctica. Otras investigaciones, precisan sobre el conocimiento didáctico-matemático (Godino, 2009). Este conocimiento deriva en un modelo que analiza y caracteriza la instrucción matemática del profesor desde el enfoque ontosemiótico del conocimiento y la instrucción matemática (Godino, Batanero y Font, 2007). También hay estudios sobre el conocimiento especializado del profesor de matemáticas el Mathematics Teacher's Specialised Knowledge, conocido como el MTSK (por sus siglas en inglés) (Carrillo et al., 2013; Carrillo et al., 2018). Este constructo compone un modelo que propone lo especializado como la conjunción de conocimientos matemáticos y didácticos

La pluralidad epistemológica se contrapone a la hegemonía en el sentido de la consideración de diferentes argumentaciones, significados y procedimientos que existen y que son asociados al conocimiento matemático en una situación y contexto específico (Cordero et al., 2015).

4 La transversalidad es la resignificación de los usos del conocimiento entre escenarios o dominios de conocimiento, por ejemplo: entre la escuela y el trabajo; o entre la matemática y la ingeniería (Cordero, Del Valle y Morales, 2019).

5 De acuerdo a la información proporcionada por el Ministerio de Educación de Chile, la educación media contempla 4 años de ciclo escolar. Es decir, atiende a jóvenes entre los 14 y 18 años. Cabe señalar que a partir del año 2027 este ciclo durará 6 años. Actualmente 900.000 jóvenes pertenecen a la educación media, los que se distribuyen en 3.471 establecimientos escolares (www.escolar.mineduc.cl/media/). 
específicos del profesor de matemáticas. Se subraya que, en estas investigaciones, una característica es preguntar por el conocimiento matemático que requiere el profesor para enseñar matemáticas. Sin embargo, hasta ahora, los resultados que nos ofrecen solo lo han enfocado al saber matemático escolar. Es decir, no lo han ampliado a otros saberes matemáticos articulados a los referentes de la escuela, del trabajo y la ciudad del que aprende para enseñar.

La identidad disciplinar es una perspectiva que tensa la problemática de la formación inicial ya que a diferencia de las investigaciones que movilizan cambios en la instrucción de los estudiantes de docencia y profesores formadores, la identidad disciplinar conlleva un cambio epistemológico, con el cual se recuperan los referentes de los saberes matemáticos que emergen en el cotidiano de la gente.

Entonces nos damos a la tarea de evidenciar empíricamente que la identidad disciplinar es un factor de la formación de los estudiantes de docencia en matemáticas. Mostramos cómo ese factor interviene en la resignificación del conocimiento matemático y en la definición de la función del docente de matemáticas. La fuente de análisis se desarrolla en el escenario escolar-académico del estudiante acompañado del desarrollo de la visión de la enseñanza y la del conocimiento matemático que va construyendo en el hábito permanente del diseño de actividades escolares. Estas últimas, por una parte, favorecen la emergencia de argumentaciones autónomas. Y, por otra, expresan la construcción de la identidad disciplinar; así como, define la función del docente de matemáticas.

\section{LOS USOS Y SIGNIFICADOS DEL CONOCIMIENTO MATEMÁTICO: UN NUEVO MARCO DE REFERENCIA}

En la enseñanza de la matemática escolar habitual predomina la centración a los objetos matemáticos. Por ejemplo, el concepto de función, límite, derivada, integral, series, entre otros. Estos conceptos en las fases educativas deben alcanzar una compresión terminal (Cordero, 2001). Sin embargo, favorecer en sí mismo estos conceptos, y procedimientos que conllevan aprenderlos, provoca la invisibilidad de ciertas categorías del conocimiento matemático, tales como la predicción, el comportamiento tendencial de las funciones, la analiticidad de las funciones (Cordero, 2008), la optimización (Cordero, Del Valle y Morales, 2019), la compensación (Medina, Ruiz y Cordero, 2019) y la anticipación (PérezOxté y Cordero, 2019) (Ver Tabla 1). 
Tabla 1. Socioepistemología del Cálculo y Análisis

\begin{tabular}{|c|c|c|c|c|c|c|}
\hline \multicolumn{7}{|c|}{ Situaciones } \\
\hline $\begin{array}{l}\text { Construcción } \\
\text { de lo } \\
\text { matemático }\end{array}$ & Variación & Transformación & Aproximación & Selección & Ponderación & Periodización \\
\hline Significaciones & $\begin{array}{c}\text { Flujo } \\
\text { Movimiento } \\
\text { Acumulación } \\
\text { Estado Permanente }\end{array}$ & $\begin{array}{l}\text { Patrones de } \\
\text { comportamiento } \\
\text { gráficos y } \\
\text { analíticos }\end{array}$ & $\begin{array}{c}\text { Limite } \\
\text { Derivación } \\
\text { Integración } \\
\text { Convergencia }\end{array}$ & $\begin{array}{l}\text { Patrón de } \\
\text { adaptación }\end{array}$ & $\begin{array}{l}\text { Distribución de } \\
\text { comportamiento }\end{array}$ & $\begin{array}{l}\text { Reproducción de } \\
\text { comportamientos }\end{array}$ \\
\hline Procedimientos & $\begin{array}{l}\text { Comparación de } \\
\text { dos estados }\end{array}$ & $\begin{array}{l}\text { Variación de } \\
\text { parámetros }\end{array}$ & Operaciones lógico formales & $\begin{array}{l}\text { Distinción de } \\
\text { cualidades }\end{array}$ & Equiparar & $\begin{array}{l}\text { Comparación de } \\
\text { periodos }\end{array}$ \\
\hline Instrumentos & $\begin{array}{c}\text { Cantidad de } \\
\text { variación continua } \\
\begin{array}{c}f(x+h)-f(x)=\alpha h \\
\alpha=f^{\prime}(x)\end{array}\end{array}$ & 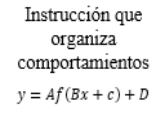 & $\begin{array}{c}\text { Formas analiticas } \\
\lim _{h \rightarrow 0} \frac{f(x+h)-f(x)}{h}=f^{\prime}(x)\end{array}$ & $\begin{array}{c}\text { Lo estable } \\
\nabla f(x)-\lambda \nabla g(x)=0\end{array}$ & $\begin{array}{c}\text { Punto de } \\
\text { equilibrio } \\
\sum_{i=1}^{n}\left(X_{i}-\bar{X}\right)=0\end{array}$ & Interpolación \\
\hline Argumentación/ & $\begin{array}{c}\text { Predicción } \\
E_{0}+\text { variación }=E_{f}\end{array}$ & $\begin{array}{l}\text { Comportamiento } \\
\text { tendencial }\end{array}$ & Analiticidad de las funciones & Optimización & Compensación & Anticipación \\
\hline Resigni & & 10 & & & & 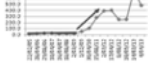 \\
\hline
\end{tabular}

Fuente. Cordero (2017).

Esas categorías son resultado de las investigaciones en socioepistemología. El principio en cada una de ellas es la descentración de los objetos terminales y el desarrollo de los procesos permanentes. Por ende, destacan los usos y significados del conocimiento matemático de la gente en su cotidiano (Cordero, 2017). De ahí que el SOLTSA asume la siguiente tarea: revelar los usos del conocimiento matemático que emergen en diferentes comunidades de conocimiento a partir de situaciones específicas que responden a los cotidianos escolares, disciplinares, profesionales u otros (Cordero, 2016a).

Se subraya que el SOLTSA se desarrolla a través de dos líneas de trabajo simultáneas: la resignificación del conocimiento matemático y su impacto educativo. En la primera se problematizan las categorías del conocimiento matemático que suceden en las comunidades entre diferentes dominios de conocimiento que obligadamente entran en juego: el dME, el campo disciplinar y el cotidiano de la comunidad. En la segunda línea se conforman los multifactores y estadios que coadyuvan a la alianza de calidad de la docencia de matemáticas (Cordero, 2016b y 2017). Los multifactores son los elementos que contribuyen a lograr un resultado pero que han estado ausentes y son necesario recuperarlos, tales como: identidad, inclusión, socialización, emancipación, empoderamiento, entre otros (Ver Figura 1). 
Figura 1. Programa Socioepistemológico Sujeto Olvidado y Transversalidad de Saberes (SOLTSA).

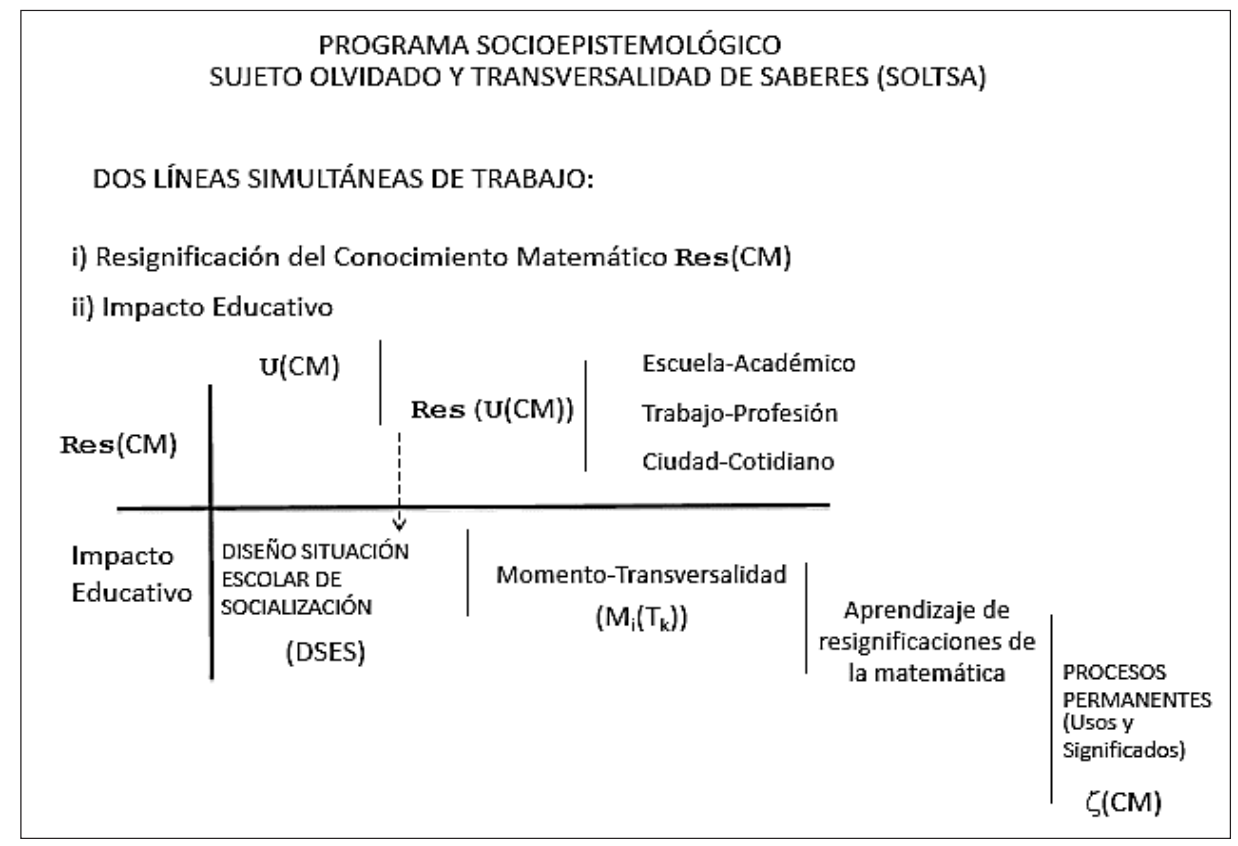

Fuente. Cordero (2017).

Lograr el impacto educativo es un gran desafío ya que esto implica un Rediseño del discurso Matemático Escolar (RdME) (Cantoral y Farfán, 2003; Cantoral, 2013), pero es más cierto cuando la primera línea coadyuva a identificar las resignificaciones que son parte del cotidiano de la gente. Sin embargo, no es fácil ya que el dME está normado por las definiciones y conceptos. Lo que transforma a la matemática escolar en un conocimiento acabado, de tipo utilitario y con un carácter hegemónico (Soto y Cantoral, 2014).

En otras palabras, el aprendizaje de la matemática escolar favorece adoptar un conocimiento matemático que no se puede transformar ni menos trastocar ya que la enseñanza no está diseñada para que esto ocurra. Al contrario, se memorizan y reproducen procedimientos y argumentos que sólo se utilizan de manera utilitaria y con cierta linealidad en problemas específicos de la matemática escolar. Por ejemplo, el estudiante de docencia de la matemática primero aprende el concepto de función, luego el de límite, derivada y posteriormente el de integral y series. Si ese estudiante no logra el cometido anterior la responsabilidad recae en él porque no pudo con las matemáticas, y en el profesor porque no enseñó de manera efectiva. De modo que el problema se focaliza sobre la persona. No se percibe que el cometido fomenta argumentos exógenos a la realidad del que aprende para enseñar y del profesor de matemáticas. Esto deriva la tensión con nuestro planteamiento, ya que la Teoría Socioepistemológica de la Matemática Educativa (TSME) asume como problemática la construcción y difusión del conocimiento matemático; despersonificando así, la responsabilidad que menoscaba la percepción del estudiante y del profesor de matemáticas (Cantoral, 2013). 
Legitimar el uso del conocimiento matemático que emerge en el escenario escolaracadémico del estudiante que aprende para enseñar, es un desafío que necesariamente se debe afrontar. Para tal fin, se debe construir un programa permanente. Esto implica valorizar el conocimiento que es propio de esta comunidad, ya que es algo que no se le ha dado para que lo adquiera; al contrario, se crean las condiciones para que emerjan los usos y significados a partir de una situación específica. Lo anterior, conlleva hacer más robusta la epistemología de usos del conocimiento matemático desde el cotidiano del estudiante de docencia de la matemática. Pero también, promueve una base de conocimiento que permite el diseño de actividades escolares donde emergen argumentaciones autónomas en los que aprenden.

\section{RESIGNIFICACIÓN DEL CONOCIMIENTO MATEMÁTICO}

Transformar la formación inicial docente demanda cambiar la epistemología dominante del dME, por lo que la función del docente se define a la luz de la identidad disciplinar que se constituye desde la pluralidad epistemológica y la transversalidad de saberes.

El cambio epistemológico requiere un programa permanente que acompañe la formación inicial docente. Un hilo conductor son las categorías del conocimiento matemático, ya que expresan un conocimiento que a priori no está en la enseñanza de la matemática escolar.

En relación con este planteamiento, destaca un programa para la formación inicial que impulsa un grupo de académicos que estudia el conocimiento matemático desde su construcción social (Soto y Silva-Crocci, en prensa). Este programa está inscrito en una institución de educación superior con experiencia y tradición en la formación inicial docente en Chile. Su plan de estudio contempla 10 semestres, lo que equivale a 47 asignaturas que deben ser aprobadas para optar al grado de Licenciado en Educación Matemática y Computación.

Este programa, para la formación inicial, señala que uno de sus objetivos es:

Fortalecer la enseñanza y aprendizaje de las matemáticas en diferentes niveles educativos y con diferentes comunidades de conocimiento a partir de articular las habilidades del pensamiento matemático que norman lo habitual de la matemática escolar y las categorías del conocimiento matemático (Soto y Silva-Crocci, en prensa).

En este objetivo sobresalen dos aspectos. El primero, la realidad educativa. En Chile las Bases Curriculares son las encargadas de ofrecer los aprendizajes comunes para los alumnos y las alumnas del país durante su trayectoria escolar (Mineduc, 2015). Para tal fin, se proponen cuatro habilidades que desarrollan el pensamiento matemático en los estudiantes de enseñanza básica y media según los lineamientos del Ministerio de Educación en Chile. Para este órgano del Estado las habilidades son las capacidades para realizar tareas y para solucionar problemas con precisión y adaptabilidad (Mineduc, 2015, p. 22). Las cuatro habilidades son: resolución de problemas, representación, modelar, argumentar y comunicar.

Ahora bien, el segundo aspecto versa sobre las categorías del conocimiento matemático. Una característica que tienen las categorías es que expresan lo útil al humano en una 
situación específica (Cordero, 2001). Por lo tanto, estas categorías son algo más robusto que una representación (de la realidad) o una aplicación matemática (a una situación real). Es decir:

Es una práctica plasmada específicamente como la argumentación de una situación en cuestión. Esa situación está compuesta de significaciones o resignificaciones con sus respectivos procedimientos: ambas se van construyendo de acuerdo con las operaciones que los participantes son capaces de hacer, con las condiciones que ellos son capaces de capturar y transformar y con los conceptos que van construyendo progresivamente (Cordero, 2016a, p. 62).

Cada situación específica se conforma por elementos secuenciales que construyen lo matemático: significaciones, procedimientos e instrumentos, lo que deriva en argumentaciones de la situación en cuestión. Por ejemplo, para la construcción de lo matemático en la situación de transformación, las significaciones son los patrones gráficos y analíticos, los procedimientos derivados de las significaciones son la variación de parámetros y los instrumentos que se derivan de las significaciones y procedimientos son las instrucciones que organizan comportamientos. Lo anterior, genera la argumentación de la situación de transformación: El comportamiento tendencial (Ver Tabla 2). Argumento que establece relaciones entre funciones y está compuesto por una colección coordinada de conceptos y situaciones del Cálculo donde se discuten aspectos globales de variación (Cordero, 1998, p. 6). Se subraya que el comportamiento tendencial es intrínseco a la gráfica y genera un desarrollo de usos de la gráfica que resignifica el conocimiento matemático (Cordero, 2001 y 2008).

Tabla 2. Construcción de lo matemático en comunidades de conocimiento matemático

\begin{tabular}{|c|c|}
\hline Construcción de lo matemático & Transformación \\
\hline Significaciones & Patrones de comportamientos gráficos y analíticos \\
\hline Procedimientos & Variación de parámetros \\
\hline Instrumentos & Instrucción que organiza comportamientos \\
\hline Argumentación / Resignificación & Comportamiento tendencial \\
& \\
\hline
\end{tabular}

Fuente. Cordero (2017). 
Es importante mencionar que la Tabla 2 es una epistemología que emerge en el cotidiano escolar, disciplinar y profesional de la gente. Sin embargo, a priori no se considera a este referente en la enseñanza de la matemática escolar (Cordero et al., 2015). Distinto es el caso del programa para la formación inicial docente que impulsa el grupo de académicos en Chile, quienes recuperan esta base de conocimiento con el objetivo de que el estudiante que se forma en la docencia de la matemática valorice otra clase de argumentaciones.

\subsection{DEFINICIÓN DE LA COMUNIDAD DE ESTUDIO}

Estar con los estudiantes de docencia de la matemática permitió conocer sus voces (Guber, 2001). Por lo que una pregunta que surgió fue, ¿conocemos el escenario donde esos estudiantes aprenden para enseñar? La pregunta nos enfocó al escenario que brinda las condiciones ad hoc para la preparación de la enseñanza de la matemática. Esto es, su escenario escolar-académico (Cordero et al., 2015).

En ese escenario escolar-académico se estudian y debaten los referentes teóricos de la Matemática Educativa, singularidad que permite conocer la escuela y sus protagonistas (profesores de matemáticas y estudiantes de diferentes niveles educativos). Pero también, la problemática de la enseñanza y aprendizaje de la matemática escolar desde referentes teóricos. Esto último, significa lo mismo que Lestón (2014) afirma al respecto:

Hablamos de referentes teóricos, en lugar de autores, ya que no nos interesa quedarnos con las personas sino con sus conceptualizaciones teóricas de la realidad de las aulas de matemática educativa. Y son esos referentes los que surgen en las producciones de los futuros docentes (p. 2045).

Es decir, las producciones de los que aprenden para enseñar son relevantes porque expresan su visión de la enseñanza y, también, del conocimiento matemático. En otras palabras, son una síntesis de los referentes teóricos que ellos utilizan para dialogar con estudiantes y profesores en sus experiencias de campo. Es importante señalar que la síntesis tiene una fuente de sentido que las identifica (Castells, 2001). Un ejemplo de esta fuente de sentido es la resignificación del conocimiento matemático (Cordero y Silva-Crocci, 2012; Opazo-Arellano, Cordero y Silva-Crocci, 2018). Para dar evidencias de este hecho decidimos metodológicamente analizar el escenario escolar-académico del estudiante de docencia acompañado del desarrollo de la visión de la enseñanza y la del conocimiento matemático que construyen en el hábito permanente del diseño de actividades escolares.

Cabe señalar que los investigadores centraron la atención en estos diseños porque expresan el encuentro entre la formación inicial docente y la realidad educativa, donde participan las aulas de matemáticas y las problemáticas de la enseñanza y aprendizaje de la matemática escolar. De ahí que los investigadores analizaron los momentos de los diseños y sus respectivas preguntas desde una estructura epistemológica. Se destaca que este esfuerzo inquisitivo permitió describir y mostrar la construcción de la fuente de sentido del estudiante de docencia de la matemática, expresión de un cambio epistemológico en el marco de la planificación y realización de la enseñanza.

En relación con lo anterior, sobresalen dos resultados del marco etnográfico que se formuló a partir de las inmersiones iniciales y profundas (Guber, 2001) con entrevistas semiestructuras realizadas a las comunidades de estudiantes de docencia de la matemática. 
Por una parte, está la construcción de diseños de actividades escolares que legitiman los usos del conocimiento matemático del que aprende desde la transversalidad de saberes en una situación específica. En este caso, se realizó un análisis documental de las producciones que resultan de dos asignaturas: Didáctica del Algebra y del Cálculo, y Fundamentos de la Educación Matemática. Estas dos asignaturas están inscritas en el programa para la formación inicial docente que proponen Soto y Silva-Crocci (en prensa). Por otra parte, están las argumentaciones autónomas que emergen en la puesta en escena de estos diseños. Para tal fin, el método consistió en revelar los usos del conocimiento matemático ${ }^{6}$ del que aprende, donde el hilo conductor del análisis se concentró en la resignificación del conocimiento matemático que sucedió en esa comunidad. Se reportan los usos del conocimiento matemático de una comunidad de estudiantes de docencia de la matemática, distinta a la que conformó el diseño de actividad escolar que se presenta a continuación. Cabe señalar que cursaban su quinto semestre y habían aprobado asignaturas como: Matemática Básica, Algebra I, II y III, y Cálculo I, II y III.

La puesta en escena del diseño se realizó con dos comunidades de conocimiento (ver Tabla 3). Cada comunidad realizó una puesta escena, en una sesión de trabajo con tiempo estimado de 120 minutos. A cada comunidad se les proporcionó el material sugerido en el diseño de actividad escolar. La comunidad (A y B) participó de manera voluntaria, aceptando video-grabar los diálogos y acciones realizadas en la puesta en escena. Cabe señalar que cada comunidad pertenecía a una universidad específica, las que desarrollan sus planes de estudios en Santiago de Chile.

Tabla 3. Comunidades de conocimiento matemático

\begin{tabular}{|c|c|c|c|c|}
\hline $\begin{array}{c}\text { Comunidad de } \\
\text { conocimiento matemático }\end{array}$ & $\begin{array}{c}\text { Número de } \\
\text { estudiantes }\end{array}$ & Mujeres: M & Hombres: H & $\begin{array}{c}\text { Semestre que } \\
\text { cursaban }\end{array}$ \\
\hline A & 2 & 1 & 1 & V \\
\hline B & 6 & 4 & 2 & V \\
\hline
\end{tabular}

Fuente. Elaboración propia.

\subsection{ANÁLISIS Y DISCUSIÓN}

Al diseño de actividad escolar que se puso en escena se le llamó la curva de la caída libre, el cual fue construido por dos estudiantes de docencia de la matemática (un hombre y una mujer). La situación en cuestión se sustentó en la variación de coeficientes en la transformación de funciones a partir de tres momentos. Momento 1: Traslaciones y cambios de pendientes. Momento 2: Comparación entre gráficas y relaciones de rapidez. Momentos 3: Simulación de comportamientos gráficos. Esta situación privilegia la

\footnotetext{
6 Los usos del conocimiento matemático se entienden como las funciones orgánicas de las situaciones (funcionamientos), que se manifiestan en las "tareas" que componen la situación. Y, la clase de esas tareas son la forma del uso del conocimiento matemático. Las tareas pueden ser: actividades, acciones, ejecuciones, alternancia de dominios propias del organismo de la situación (Cordero y Flores, 2007).
} 
concepción de función desde una relación con su comportamiento. La función se entiende como una relación entre parámetros que representa la variación de cada uno de ellos, es decir: la forma de comportarse respecto a los otros parámetros. Se afirma que la función se resignifica como una instrucción que organiza comportamientos donde $f$ se transforma en $F=A f(B x+C)+D$ (Buendía y Cordero, 2005; Cordero, 1998 y 2001).

Para exhibir la situación, la curva de la caída libre, a continuación se presentan los momentos y las preguntas que favorecen la emergencia de los usos del conocimiento matemático del que aprende (ver Tabla 4).

Tabla 4. Momentos de la curva de la caída libre

\begin{tabular}{|c|c|} 
La curva de la caída libre: \\
Apila una cierta cantidad de libros en la cual se sostenga un lado de un pedazo \\
de cartón piedra formando una rampa con cierto grado de inclinación. Mide el \\
ángulo de inclinación de la rampa con el transportador y luego mide el tiempo \\
en que se demora en caer la pelota hasta que llega al final de la rampa.
\end{tabular}

Fuente. Diseño de estudiantes de docencia de la matemática. 


\subsubsection{Resultados de la puesta en escena}

A continuación, se describen los resultados de la puesta en escena del diseño de actividad escolar. Para tal fin, se presentan las Tablas 5, 7 y 9. En cada una de ellas se acentúan los aspectos más relevantes. Por ejemplo, la Tabla 5 exhibe el síntoma del uso de lo cuadrático desde su funcionamiento y forma. Esto es la reproducción y comparación de trayectorias a partir de dibujos y descripciones alusivas al comportamiento cuadrático que denota la situación en cuestión.

En lo anterior, se significan parámetros analíticos y gráficos desde el análisis de la posición del móvil (inicial, intermedia o final) en la rampa. Por lo que, en el primer momento del diseño de actividad escolar, la comunidad de conocimiento matemático (A) valorizó cómo se mueve un móvil. En este contexto, el síntoma se expresa en la utilización de flechas y comillas. Lo que distingue la dirección que lleva el móvil sobre el plano inclinado desde los referentes de la escuela, del trabajo y de la vida que son propios de esta comunidad de conocimiento (ver en Tabla 5: A4 y A2 respectivamente). A1 respecto, algunas de las afirmaciones de esta comunidad son: "Que la pelota va en esa dirección" y "la pelota descendiendo con una especie de comillas que simbolizan este movimiento" (ver en Tabla ${ }^{7} 6$ transcripción del audio). Se subraya que la dirección del móvil demanda significar traslaciones y cambios de pendientes en los comportamientos cuadráticos.

Otro aspecto que conviene señalar de la Tabla 5, respecto al primer momento del diseño de actividad escolar, es la temporalidad $\left(\mathrm{t}_{\mathrm{i}}\right)$. La que coadyuva a describir la posición del móvil a partir de una secuencia (temporal) en la tarea de hacer descender o ascender el móvil por el plano inclinado. Al respecto, la comunidad (A) señaló lo siguiente: "dibujar la pelota en el inicio del movimiento, digamos en una especie de instante cero" $y$ "va agitando el aire digamos en un instante distinto al inicial" (ver en Tabla 5: A1, A2 y A3. Y, ver en Tabla 6 transcripción del audio).

Tabla 5. El síntoma del uso de lo cuadrático

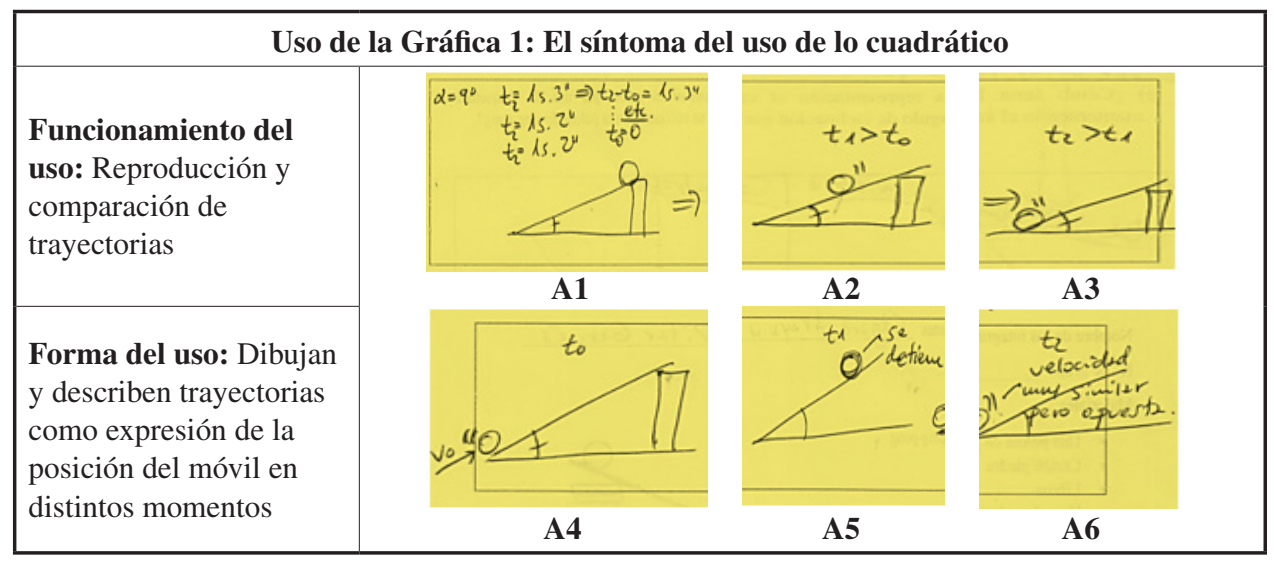

Fuente. Respuestas de estudiantes de docencia de la matemática.

En la entrevista realizada, se identificó al entrevistador por el código: (E). Y, a los integrantes de la comunidad de conocimiento matemático (A): mujer (M1) y hombre (H1). 
Tabla 6. Utilización de comillas y flechas en la descripción del movimiento

\begin{tabular}{|c|l|}
\hline Código & \\
\hline & \multicolumn{1}{|c|}{ Preguntas y afirmaciones } \\
E & ¿Cómo representarías el movimiento de la pelota? \\
M1 & Pi fuera un dibujo, ¿cuál podría ser? \\
E & Em. \\
M1 & O, ¿cuántas partes? ¡No sé! \\
H1 & Es que podría ser en tres partes o puede ser el dibujo con una flechita como que \\
M1 & avanza. \\
E & Ya, ¿a ver?, ¿cómo quedaría? \\
E & ¿Por qué la flecha? \\
M1 & Em. \\
E & ¿Qué representa para ti la fecha? \\
M1 & Que la pelota va en esa dirección. \\
E & Ya, ok. \\
E & H1, tú decías que se puede hacer de varias maneras; ¿De qué otra manera lo harías? \\
H1 & Otra manera en la que a mí se me ocurriría, sería en varios momentos. Por decirlo \\
E & así. \\
H1 & Entonces, ¿cómo quedaría? \\
& Al principio dibujar la pelota en el inicio del movimiento, digamos en una especie de \\
& instante cero con el mismo ángulo en este caso. En un segundo instante posterior, \\
E & hacer la misma situación, pero ya la pelota descendiendo con una especie de \\
M1 & comillas que simbolizan este movimiento. \\
& ¿Por qué son importantes las comillas? \\
& Porque en este sentido, representa el movimiento del objeto de donde viene es al \\
& revés que la fecha. De donde viene simboliza digamos así, va agitando el aire digamos \\
E & en un instante distinto al inicial. Y, el tercer instante sería cuando ya la pelota está \\
M1 & llegando al suelo o está en el suelo. Lo que sería un instante posterior al anterior. \\
E & Ok. M1 decía que se podía de otra manera. Entonces, ¿cómo sería? \\
M1 & [..] No sé, la expresión sería como de la velocidad o la posición de la pelota. \\
& Ya. [...], pero ¿cómo sería la expresión? \\
& iNo sé, la verdad no séi \\
\hline
\end{tabular}

Fuente. Respuestas de estudiantes de docencia de la matemática.

Lo icónico y verbal, dos argumentaciones soslayadas en lo habitual de la matemática escolar, desde la reproducción y comparación de trayectorias, confrontan el ejemplo E1 que propone el Mineduc (2016) (ver Tabla 11) ${ }^{8}$. La propuesta que impulsa el Ministerio de Educación en Chile por intermedio del Programa de Estudio para Segundo año Medio se contextualiza en el lanzamiento de la bala donde el procedimiento es reemplazar en la expresión analítica valores específicos en la variable independiente. En ese caso, el objetivo es determinar la posición del móvil en un tiempo señalado. La localidad de ese

La Tabla 11 es un resumen de los objetivos de aprendizajes, los indicadores de evaluación y tres de los diez ejemplos propuestos en el Programa de Estudios de Segundo año Medio sobre la función cuadrática en el eje de álgebra y funciones (Mineduc, 2016). La Tabla 11 tiene el objetivo de confrontar las argumentaciones que emergen producto de los diseños de actividades escolares y la enseñanza de la matemática escolar que promueve el Ministerio de Educación en Chile. 
procedimiento hegemoniza el tiempo como una variable independiente en la enseñanza habitual de la matemática escolar. Así como, la unión de puntos en el plano cartesiano a partir de la construcción de tablas numéricas. Estos argumentos, externos a la construcción social del conocimiento matemático, impiden el desarrollo de argumentaciones como: la dirección y la temporalidad en la descripción del movimiento. Zaldívar y Briceño (2019), se refieren a este tipo de argumentaciones como formas culturales de saberes. Las que son propias de los que participan en la situación en cuestión.

Ahora bien, la Tabla 7 muestra el uso de lo cuadrático a partir de la reproducción y comparación de comportamientos en intervalos acotados. En este sentido, la comunidad de conocimiento matemático (A) valorizó la rapidez del móvil con base en el análisis de las gráficas cartesianas que aluden a distancias recorridas en tiempos específicos. La discusión, por lo tanto, se enfocó en el análisis de comportamientos crecientes o decrecientes en intervalos acotados; distinguiendo así aspectos locales en la gráfica de la función.

Se subraya que la trayectoria del móvil, en el segundo momento del diseño de actividad escolar, se resignifica en la reproducción y comparación de comportamientos cuadráticos a la luz de la clase de tareas que experimenta esta comunidad de conocimiento (ver en la Tabla 7: A7 y A8). En este caso, la clase de tarea se enfocó en el análisis de gráficas que resultan de variar el grado de inclinación del plano inclinado $\left(0^{\circ}, 45^{\circ}\right.$ y $\left.90^{\circ}\right)$. Esto implica variar parámetros de la función en ausencia de expresiones analíticas en la situación en cuestión. Es importante señalar, en este contexto, un aspecto insoslayable. Esto es, la experimentación ya que identifica la cualidad de lo cuadrático respecto de comportamientos lineales.

Otra resignificación en el segundo momento del diseño de actividad escolar es el uso de la temporalidad. En el primer momento, la temporalidad ayudó a identificar la dirección del móvil en el plano inclinado cuando se describe su posición. Sin embargo, en el segundo momento, la temporalidad denotada por $\left(\mathrm{t}_{\mathrm{i}}\right)$ ayudó a describir qué tan rápido crece o decrece el comportamiento cuadrático en intervalos de tiempos acotados (los cuales no necesariamente son numéricos). Para esto último, la comunidad de conocimiento matemático (A) bosquejó líneas verticales y horizontales sobre el plano cartesiano con el objetivo de analizar comportamientos locales en la gráfica (ver en Tabla 7: A8 y A9). En este sentido, destaca la identificación de puntos máximos o mínimos en la gráfica. Por lo que, la temporalidad permitió a la comunidad de conocimiento matemático (A) caracterizar cualitativamente el comportamiento de un móvil a partir de $\mathrm{t}_{0}, \mathrm{t}_{1} \mathrm{y}_{2}$. Lo que deriva, por ejemplo, en que $t_{1}$ se significó como un máximo en la gráfica (ver en Tabla 7: A10. Y, ver en Tabla 8 transcripción de audio). 
Tabla 7. El uso de lo cuadrático

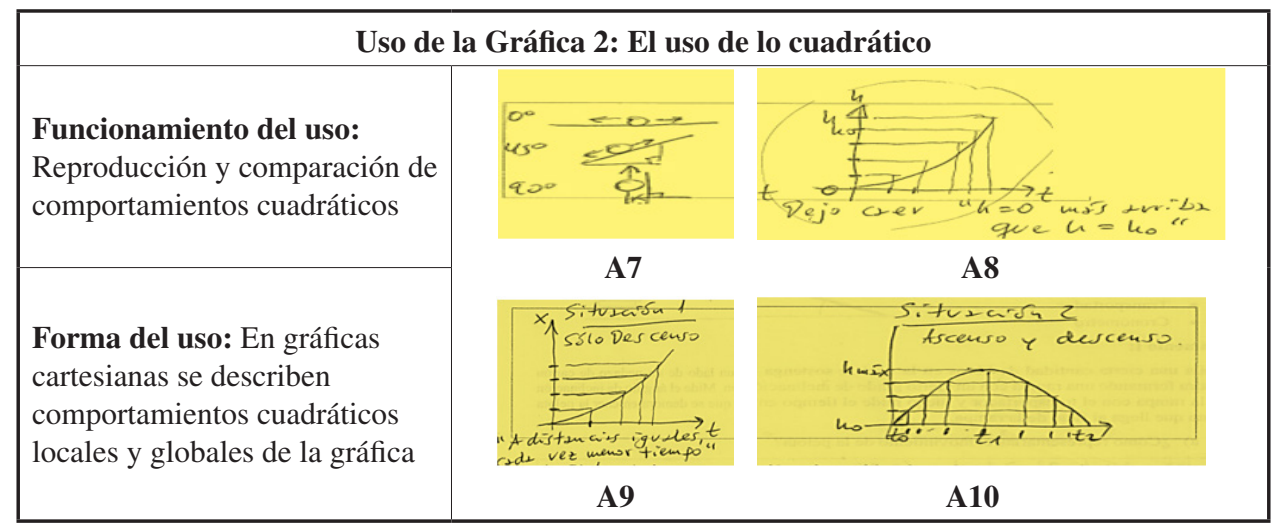

Fuente. Respuestas de estudiantes de docencia de la matemática.

Tabla 8. Análisis de intervalos acotados y definición de puntos máximos o mínimos

\begin{tabular}{|c|c|}
\hline Código & Preguntas y afirmaciones \\
\hline H1 & $\begin{array}{l}\text { En este caso, em }[\ldots] \text { es que son dos casos distintos. [...] Entonces, ésta sería la } \\
\text { situación } 1 \text { (ver en Tabla 6: A9). Sólo descenso. Habría que aclarar que los positivos } \\
\text { están hacía abajo y que el movimiento sería algo así, cada vez los intervalitos son cada } \\
\text { vez mayores y el tiempo es menor. }\end{array}$ \\
\hline $\mathbf{E}$ & Entonces, para entender, ¿cómo quedarían los intervalos? \\
\hline M1 & $\begin{array}{l}\text { En realidad, tendrían que ser con distancias iguales, entonces al tener intervalos con } \\
\text { distancias iguales tendríamos intervalos de tiempo cada vez más pequeños. } \\
\text { Entonces, } \mathrm{H} 1 \text {, ¿en distancias iguales la pelota va a tardar menos en caer? }\end{array}$ \\
\hline $\mathbf{E}$ & ¡Claro; \\
\hline M1 & Y, ¿qué pasa en la situación dos? \\
\hline $\mathbf{E}$ & $\begin{array}{l}\text { Es literalmente partiendo desde abajo, sube y luego cae de nuevo. En ese caso, la } \\
\text { situación sería. [...] Bueno, lo otro es que aquí yo le estoy dando una velocidad inicial. }\end{array}$ \\
\hline M1 & $\begin{array}{l}\text { Entonces, al tener una velocidad inicial esto trae una pendiente distinta de cero. Así } \\
\text { que este movimiento alcanzaría la altura máxima y después volvería a caer de la misma } \\
\text { manera. Entonces, está sería la altura máxima }\left(\mathbf{t}_{1}\right) \text {, la otra sería la altura inicial }\left(\mathbf{t}_{\mathbf{0}}\right) \text {. } \\
\text { Y, aquí estaría el }(\mathbf{t}) \text {; tiempo final. (Ver en Tabla 6: A10). }\end{array}$ \\
\hline
\end{tabular}

Fuente. Respuestas de estudiantes de docencia de la matemática.

La reproducción y comparación de comportamientos cuadráticos desde el análisis local de la gráfica confronta el ejemplo E2 presente en la Tabla 11, ya que mientras el objetivo de E2 es el tránsito entre un registro analítico y uno tabular y, también, uno gráfico; el diseño de actividad escolar, en el segundo momento, valoró qué tan rápido crece o decrece un comportamiento cuadrático. Para tal fin, la comunidad de conocimiento matemático (A) puso en uso la temporalidad a partir del análisis de intervalos de tiempos acotados; lo que 
distingue máximos y mínimos en la gráfica. La tensión está en que, para E2 la gráfica de la función se inscribe en una secuencia que sólo tiene lugar en la enseñanza habitual de la matemática escolar. Soslayando, por ejemplo, el estudio local de la gráfica en el plano cartesiano (Briceño y Buendía, 2016).

Por último, se analiza la Tabla 9. En ella se describe el uso del análisis de comportamientos cuadráticos en la comunidad de conocimiento matemático (A). En este tercer momento del diseño de la actividad escolar, se destaca la simulación de comportamientos gráficos. Es decir, se promueven gráficas específicas donde su comportamiento distingue el contexto que las produce (ver en Tabla 9: A11, A13 y A15). Un resultado en esta comunidad de conocimiento es dar valor de uso a: modelar comportamientos cuadráticos a partir de analizar, comparar e interpretar comportamientos tendenciales de la función. En este contexto, la función es la que organiza comportamientos. En palabras de Cordero (2001) esto es lo útil al humano. Una expresión de lo anterior es la anticipación de comportamientos gráficos, lo que resulta del análisis local y global de la gráfica.

Un ejemplo es el bosquejo A12 presente en la Tabla 9. En este caso la comunidad de conocimiento matemático (A) analizó el comportamiento local de la gráfica presente en A11, lo que determinó simultáneamente el comportamiento global del móvil sobre el plano inclinado. El análisis local se distingue en A12 cuando señala: "el objeto sube, sube, hasta que finalmente se detiene" (ver en Tabla 10 transcripción de audio).

Otro ejemplo donde está presente el análisis local y global de la gráfica de la función es el bosquejo A14 presente en la Tabla 9. En este sentido, la comunidad de conocimiento señala: "el gráfico tiene un tramo constante al principio. Después se observa un aumento de la pendiente y un quiebre, hay una punta ahí". Pero, también cuando señala: "el objeto va con una velocidad muy grande y de repente cero" (ver en Tabla 10 transcripción de audio).

Es importante, también, analizar el bosquejo A16 de la Tabla 9. Cabe señalar que la gráfica A15 organiza comportamientos cuadráticos crecientes, decrecientes o ninguno de ellos. En este contexto, emergen los usos del conocimiento matemático desde el análisis de comportamientos cuadráticos de la gráfica. Por ejemplo, con expresiones como: "al principio tiene un tramito constante" o "después, su pendiente aumenta mucho". Para determinar cómo se mueve y qué rapidez tiene, es fundamental dimensionar la trayectoria del móvil y la temporalidad. Por lo tanto se resignifica el uso de la gráfica, ya que la comunidad de conocimiento matemático modela un comportamiento gráfico global desde el estudio local de la gráfica donde hay cambios de pendientes y, también, máximos y mínimos en la gráfica (ver en Tabla 10 transcripción de audio). 
Tabla 9. Análisis de comportamientos cuadráticos

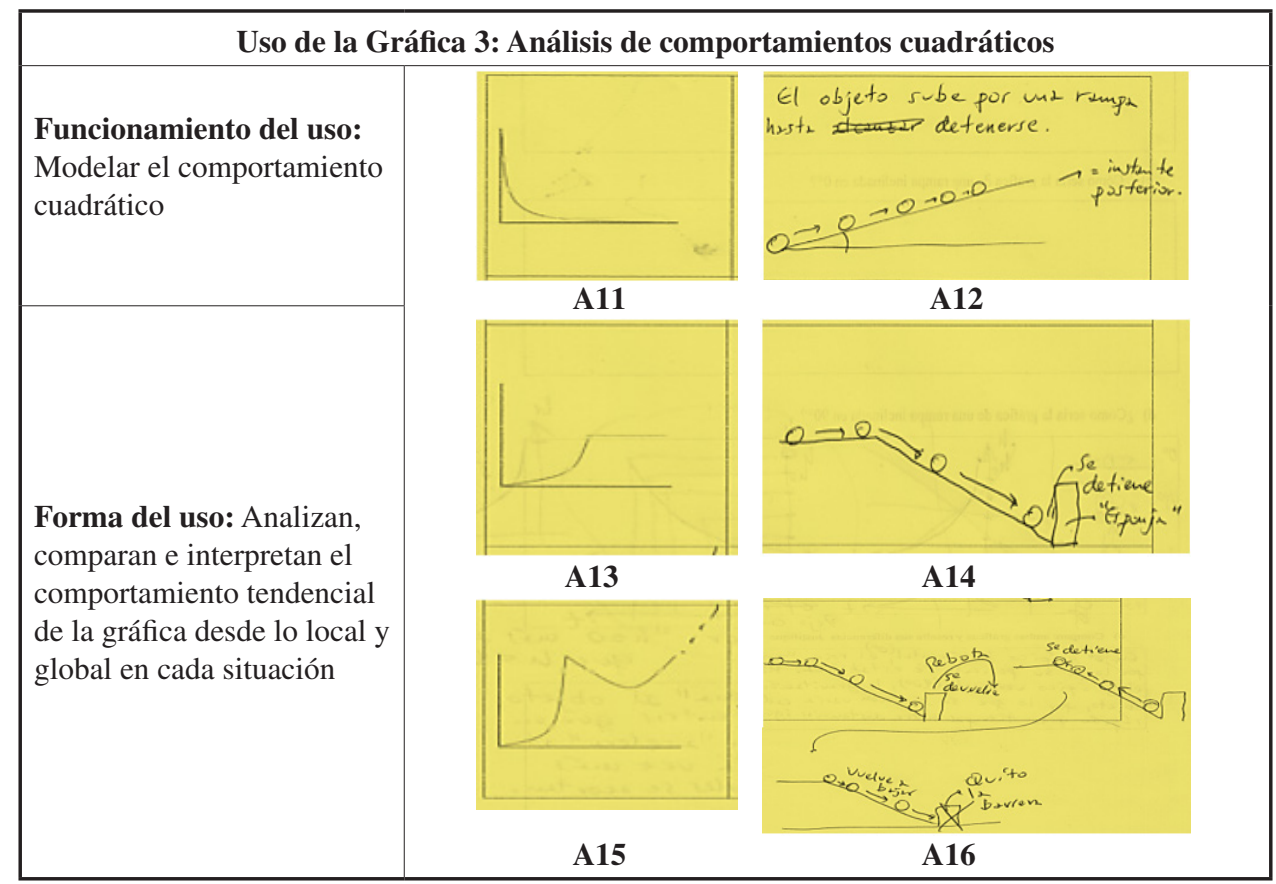

Fuente. Respuestas de estudiantes de docencia de la matemática. 
Tabla 10. Anticipación de comportamientos gráficos en la situación en cuestión

\begin{tabular}{|c|c|}
\hline Código & Preguntas y afirmaciones \\
\hline $\begin{array}{c}E \\
H 1\end{array}$ & $\begin{array}{l}\text { Ok. Entonces, en el primer caso H1 (ver en Tabla 7: A11) ¿Cuál es tu descripción? } \\
\text { Mi propuesta es que, como el objeto digámoslo viene con una velocidad muy grande, } \\
\text { digamos negativa pero muy grande, es como si el objeto estuviera ascendiendo por una } \\
\text { rampa. Entonces, el objeto al ascender por esta rampa va con una velocidad muy } \\
\text { grande y esa velocidad va disminuyendo en el sentido que en este gráfico va } \\
\text { disminuyendo su pendiente, es negativa, pero va disminuyendo. Disminuyendo su } \\
\text { valor absoluto, a eso me refiero. Entonces, el objeto sube, sube, hasta que finalmente } \\
\text { se detiene disminuyendo su velocidad cada vez más (ver en Tabla 7: A12). } \\
\text { Ok. Ahora, ¿qué pasa con el segundo caso? (ver en Tabla 7: A13). }\end{array}$ \\
\hline $\begin{array}{c}\mathbf{E} \\
\mathrm{H} 1\end{array}$ & $\begin{array}{l}\text { En la segunda, el gráfico tiene un tramo constante al principio. Después se observa } \\
\text { un aumento de la pendiente y un quiebre, hay una punta ahí. } \\
\text { Ok. Pero ¿qué pasa luego? }\end{array}$ \\
\hline $\begin{array}{c}\mathbf{E} \\
\mathbf{H} 1\end{array}$ & $\begin{array}{l}\text { Pasa que, en esa punta, la pendiente se hace horizontal; se hace cero. Entonces, el } \\
\text { objeto para ir en un movimiento constante va por un plano con una cierta velocidad } \\
\text { inicial. Al producirse este aumento el objeto cae por un plano inclinado y, después, llega } \\
\text { a una muralla donde choca y se queda ahí. } \\
\text { Entonces, ¿ahí se detiene? }\end{array}$ \\
\hline $\begin{array}{c}\mathbf{E} \\
\mathbf{H} 1\end{array}$ & $\begin{array}{l}\text { Claro, pasa que literalmente el objeto va con una velocidad muy grande y de repente } \\
\text { cero. Que es un quiebre en la naturaleza digámoslo así porque no hay una continuidad, } \\
\text { no hay algo suave, es una ruptura, un choque. } \\
\text { Y, ¿qué pasa en el último caso? (ver en Tabla } 7 \text { : A15). }\end{array}$ \\
\hline $\begin{array}{c}E \\
\text { H1 }\end{array}$ & $\begin{array}{l}\text { En el último, es algo parecido. Pero, al principio tiene un tramito constante. Después, } \\
\text { su pendiente aumenta mucho. Y, también hay otra punta. Pero la diferencia es que acá } \\
\text { el resultado de ese quiebre no es que la pendiente sea horizontal, sino que se hace } \\
\text { negativa. De ser positiva pasa a ser negativa. Entonces, significa que el objeto si va } \\
\text { avanzando en una cierta dirección choca con este obstáculo y se devuelve. Se mueve con } \\
\text { sentido opuesto y con velocidad menor en este caso. Entonces, rebota, se devuelve por } \\
\text { el plano inclinado y al final se termina deteniendo porque en un momento la gráfica } \\
\text { tiene un mínimo digamos así local. Y después, comienza a aumentar de nuevo por lo } \\
\text { tanto se empieza a devolver de nuevo por el plano, luego ya no sabemos qué pasa. Lo } \\
\text { más probable es que si quito la barrera, siga cayendo. }\end{array}$ \\
\hline
\end{tabular}

Fuente. Respuestas de los estudiantes de docencia de la matemática.

El comportamiento tendencial de las funciones se expresa en la modelación del comportamiento que exige la simulación en cuestión. Esto confronta la reproducción de la gráfica presente en el ejemplo E3 de la Tabla 11. Para el primer caso, se valorizó el estudio y análisis de comportamientos locales que anticipan el comportamiento global de la gráfica. En el segundo caso, primero se completa una tabla a partir de $\mathrm{h}=\frac{1}{20} \mathrm{v}_{0}^{2}$. Luego, se elabora la gráfica a partir de los puntos específicos que se determinan. Y, finalmente, se señala en el gráfico un punto $(\mathrm{x}, \mathrm{y})$. El primer caso valoriza los usos del conocimiento del que aprende en su cotidiano. El segundo caso, dispone adquirir y reproducir un conocimiento matemático que se utiliza preferentemente en problemas específicos de la matemática escolar. 
Tabla 11. Programa de estudio de Segundo año Medio

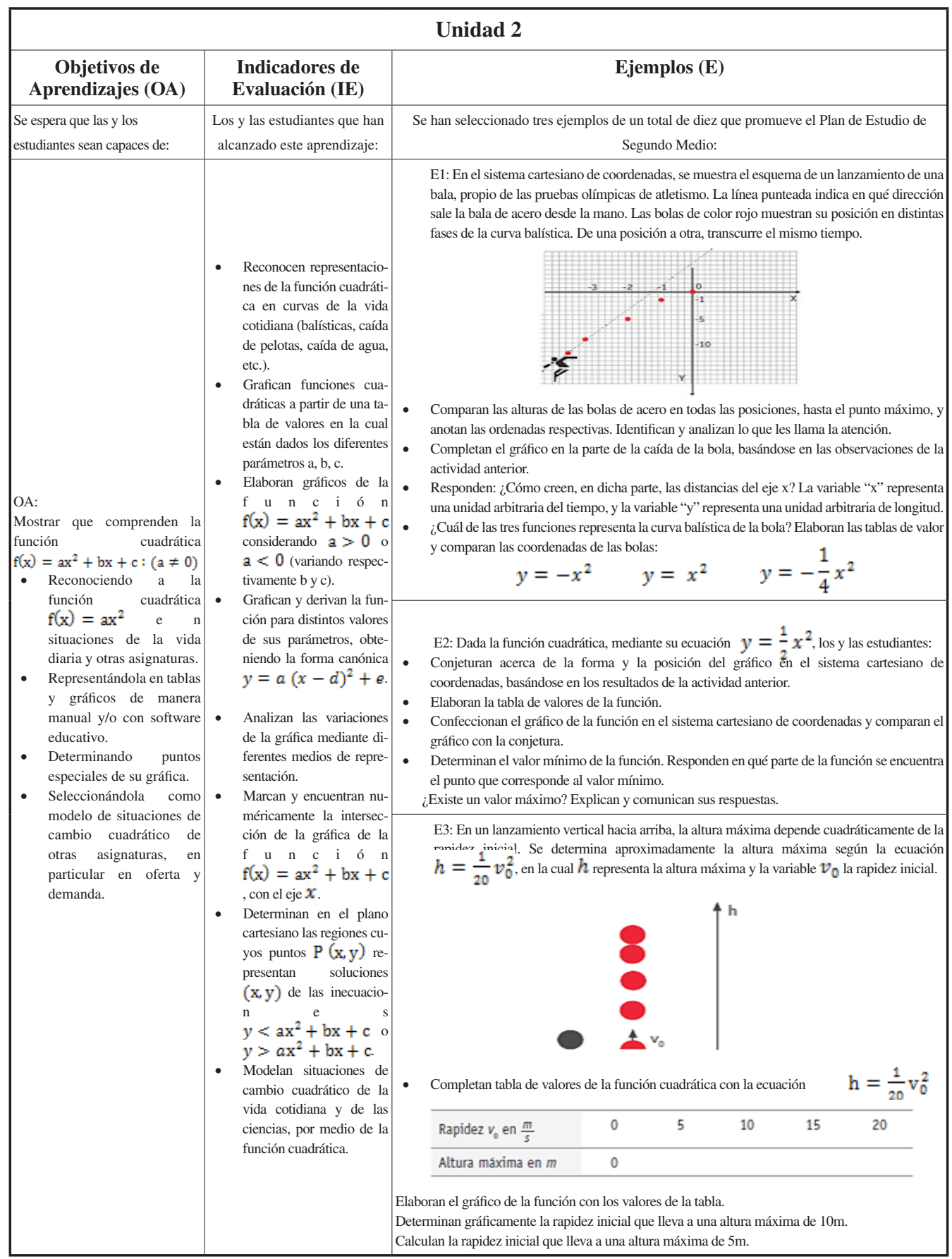

Fuente. (Mineduc, 2016). 
Las Tablas 5, 7 y 9 muestran las argumentaciones que emergen producto de la puesta en escena del diseño de actividad escolar. En ese sentido, los usos del conocimiento matemático que se exhiben valorizan la pluralidad epistemológica. Pero también, la transversalidad de saberes que provoca el diseño en cuestión.

Es importante mencionar que cada momento del diseño confrontó lo habitual de la enseñanza de la matemática escolar, es decir: la epistemología dominante del dME. La confrontación, en el diseño de actividad escolar, está en la construcción de un patrón gráfico. Lo que a la comunidad de conocimiento matemático (A) permitió la emergencia del comportamiento tendencial de las funciones. Esto provoca una tensión con la matemática escolar, ya que la enseñanza habitual de la función cuadrática centra la atención en el cambio de registro. Esto es transitar de un registro analítico a uno tabular, pero también a uno gráfico (Ver Tabla 11). Contrario a este planteamiento, la construcción de un patrón gráfico legitima el comportamiento con tendencia. Para el primer caso, la gráfica es una representación de un fenómeno que se contextualiza y lleva al aula como un objeto terminal. Sin embargo, en el segundo caso, la gráfica es una argumentación en sí misma ya que soporta el desarrollo de usos del conocimiento matemático (Cordero, 2001 y 2008).

Con base a lo descrito se puede señalar lo siguiente. La identidad disciplinar es un factor que trastoca la formación inicial docente, ya que los que aprenden para enseñar legitiman un conocimiento matemático como, por ejemplo, el comportamiento tendencial de las funciones; base epistemológica que sustenta el diseño de la curva de la caída libre. Ahora bien, la legitimidad deriva en resistencia. Por lo que la visión de la enseñanza y del conocimiento matemático se pone en uso a la luz de una nueva estructura: variación de coeficientes en la transformación de funciones; hilo conductor del diseño de actividad escolar. Pero, simultáneamente, la resistencia proyecta una resignificación. Resultado de crear y mantener diseños de actividades escolares como la curva de la caída libre donde emergen los usos del conocimiento matemático del que aprende: la trayectoria del móvil y la temporalidad. Lo que se resignifica en la modelación de un comportamiento cuadrático desde el análisis local y global de la gráfica que provee la situación en cuestión.

\section{CONCLUSIONES}

Conocer a los estudiantes de docencia de la matemática demandó hacer permanentemente inmersiones en su escenario escolar-académico. Lo que permitió conocer el hábito de hacer diseños de actividades escolares, cuya base es la resignificación del conocimiento matemático (fuente de sentido). Misma que emerge en las argumentaciones autónomas del que aprende como resultado de los momentos de transversalidad de saberes.

Ahora bien, los diseños de actividades escolares expresan (son las voces de) los referentes teóricos que los estudiantes de docencia utilizan en la construcción de su visión de la enseñanza y del conocimiento matemático. Esta visión se intercambia y confronta con los actores del sistema educativo. En ese sentido destaca la Matemática Educativa, ya que apoya y sustenta el proceso que exige preparar la enseñanza de la matemática. Esto último, define la función del docente de matemáticas.

La identidad disciplinar desde la Teoría Socioepistemológica de la Matemática Educativa es coherente a una problemática específica: la adherencia a la epistemología dominante del dME. La tarea es evitar adoptar esta epistemología, para tal fin el cambio 
epistemológico es fundamental. Aquí está la diferencia con otros planteamientos sobre la formación inicial docente.

Para el cambio epistemológico será necesario conformar programas permanentes, de tal forma de revelar los usos del conocimiento matemático de la gente con el objetivo de conformar nuevas categorías del cocimiento matemático. Pero también, crear las condiciones (como el factor de identidad disciplinar) para que el estudiante de docencia problematice el saber matemático. Como resultado de este planteamiento conllevará tendencias donde los cuestionamientos no estarán centrados en preguntas como ¿Qué queremos que aprendan las y los estudiantes durante este año? (Mineduc, 2016, p. 22), pero si en preguntas como: ¿Cómo usa el conocimiento matemático el que aprende? (OpazoArellano, Cordero y Silva-Crocci, 2018). Esto abrirá brechas para una transformación educativa de la matemática.

\section{REFERENCIAS BIBLIOGRÁFICAS}

Ball, D. L., Thames, M.H. y Phelps, G. (2008). Content Knowledge for Teaching: What Makes it Special? Journal of Teacher Education 59(5), 389-407.

Briceño, O. y Buendía, G. (2016). Una secuencia para la introducción de la función cuadrática a través de la resignificación de aspectos variacionales. Tecné, episteme y didaxis: revista de la Facultad de Ciencia y Tecnología, 39, 111-130. Última visita 1 de agosto del 2019. Recuperado desde https://revistas.pedagogica.edu.co/index.php/TED/article/view/4584/3775

Buendía, G. y Cordero, F. (2005). Prediction and the periodical aspect as generators of knowledge in a social practice framework. A socioepistemological study. Educational Studies in Mathematics, 58(3), 299-333.

Cantoral, R. y Farfán, R. M. (2003). Matemática Educativa: una visión de su evolución. Revista Latinoamericana de Investigación en Matemática Educativa, 6(1), 27-40.

Cantoral, R. (2013). Teoría socioepistemológica de la matemática educativa. Estudios sobre la construcción social del conocimiento. Barcelona, España: Gedisa.

Carrillo, J., Flores, E., Climent, N., Contreras, L., Aguilar, Á., Escudero, D. y Montes, M. (2013). Investigación sobre el profesor de matemáticas en la universidad de Huelva (España). En C. Dolores, M. García, J. Hernández, L. Sosa (Eds). Matemática Educativa: La formación de profesores (99-119). México, D. F.: Díaz Santos.

Carrillo, J., Climet, N., Montes, M., Contreras, L., Flores-Medrano, E., Escudero-Ávila, D., Vasco, D., Rojas, N., Flores, P., Aguilar- González, A., Ribeiro, M. y Muñoz-Catalán, M. (2018). The Mathematics Teacher's Specialised Knowledge (MTK). Research in Mathematics Education, 236-253. doi: 10.1080/14794802.1479981

Castells, M. (2001). La era de la información: Economía, sociedad y cultura. Volumen II: El poder de la identidad. Buenos Aires, Argentina: Siglo XXI.

Cordero, F., Del Valle, T. y Morales, A. (2019). Usos de la optimización de ingenieros en formación: el rol de la ingeniería mecatrónica y de la obra de Lagrange. Revista Latinoamericana de Investigación en Matemática Educativa, 22(2), 185-212. doi: 10.12802/relime.19.2223

Cordero, F. (2017). La matemática y lo matemático. Transversalidad y modelación: un programa socioepistemológico. Manuscrito en preparación.

(2016a). Modelación, funcionalidad y multidisciplinaridad: el eslabón de la matemática y el cotidiano. En J. Arrieta y L. Díaz (Eds.), Investigaciones latinoamericanas de modelación de la matemática educativa (pp. 59-88). Barcelona, España: Gedisa.

(2016b). La función social del docente de matemáticas: pluralidad, transversalidad y reciprocidad. En S. Estrella, M. Goizueta, C. Guerrero, A. Mena, E. Montoya, A. Morales, 
M. Parraguez, E. Ramos, P. Vásquez y D. Zakaryan (Eds). XX actas Jornadas Nacionales de Educación Matemática (pp. 23-30), ISSN 0719-8159. Valparaíso, Chile:_SOCHIEM, IMAPUCV. Última visita 17 de octubre del 2017. Recuperado de http://ima.ucv.cl/xxjnem

Cordero, F., Gómez, K., Silva-Crocci, H. y Soto, D. (2015). El discurso matemático escolar: la adherencia, la exclusión y la opacidad. Barcelona, España: Gedisa.

Cordero, F. y Silva-Crocci, H. (2012). Matemática Educativa, Identidad y Latinoamérica: El quehacer y la usanza del conocimiento disciplinar. Revista Latinoamericana de Investigación en Matemática Educativa, 15(3), 295-318.

Cordero, F. (2008). El uso de las gráficas en el discurso del cálculo escolar. Una visión socioepistemológica. En R. Cantoral, O. Covián, R. M. Farfán, J. Lezama \& A. Romo (Eds), Investigaciones sobre enseñanza y aprendizaje de las matemáticas: Un reporte Iberoamericano (pp. 285-309). México, D. F.: Díaz de Santos-Comité Latinoamericano de Matemática Educativa. A. C.

Cordero, F. y Flores, R. (2007). El uso de las gráficas en el discurso matemático escolar. Un estudio socioepistemológico en el nivel básico a través de los libros de texto. Revista Latinoamericana de Investigación en Matemática Educativa, 10(1), 7-38.

Cordero, F. (2001). La distinción entre construcciones del cálculo. Una epistemología a través de la actividad humana. Revista Latinoamericana de Investigación en Matemática Educativa, 4(2), 103-128.

(1998). El entendimiento de algunas categorías del conocimiento del cálculo y análisis: el caso del comportamiento tendencial de las funciones. Revista Latinoamericana de Investigación en Matemática Educativa, 2(1), 56-74.

Godino, J., Batanero, C., y Font, V. (2007). The onto-semiotic approach to research in mathematics education. ZDM. The International Journal on Mathematics Education, 39(1), 127-135.

Godino, J. (2009). Categorías de análisis de los conocimientos del profesor de matemáticas. Revista Iberoamericana de Educación Matemática, 20, 13-31.

Godino, J., Giacomone, B., Batanero, C. y Font, V. (2017). Enfoque ontosemiótico de los conocimientos y competencias del profesor de matemáticas. Bolema-Boletim de Educação matemática, 31(57), 90-113.

Guber, R. (2001). La etnografía. Método, Campo y Reflexividad. Grupo Editorial Norma: Bogotá.

Lestón, P. (2014). El rol de las publicaciones en la construcción de la profesionalización docente. En Lestón, Patricia (Ed.). Acta Latinoamericana de Matemática Educativa, 27 (pp. 2043-2052). México, D.F.: Comité Latinoamericano de Matemática Educativa.

Medina, D., Ruiz, B. y Cordero, F. (2019). The argument of compensation in mathematics teacher: arithmetic mean, equilibrium point and graphs of data. Enviado para publicar.

Mendoza-Higuera, E., Cordero, F., Solís, M. y Gómez, K. (2018). El uso del conocimiento matemático en las comunidades de ingenieros. Del objeto a la funcionalidad matemática. Bolema- Boletim de Educação matemática, 32(62), 1219-1243.

Ministerio de Educación. (2016). Programa de Estudio de Segundo Medio. Matemática. Santiago, Chile. ISBN9789562926058. Última visita 15 de junio del 2018. Recuperado de https://www. curriculumnacional.cl/614/w3-propertyvalue-77584.html

. (2015). Bases Curriculares $7^{\circ} a 2^{\circ}$ medio. Santiago, Chile. ISBN9789562925815. Última visita 20 de mayo del 2017. Recuperado de https://www.curriculumnacional.cl/614/w3article-34949.html

. (2012). Estándares orientadores para carreras de pedagogía en educación media. Lom. Santiago, Chile. Última visita 5 de enero del 2015. Recuperado de https://www.cpeip.cl/ estandares-orientadores-para-la-formacion-inicial-docente/

. (2005). Informe comisión sobre formación inicial docente. Santiago, Chile. Última visita 13 de junio del 2016. Recuperado de https://dds.cepal.org/redesoc/publicacion?id=430 . (2001). Estándares de Desempeño para la Formación Inicial Docente. Santiago, Chile. 
Opazo-Arellano, C., Cordero, F. y Silva-Crocci, H. (2019). La Formación del Futuro Profesor de Matemáticas y la Construcción de la Identidad Disciplinar. En Flores, R. (Ed). Acta Latinoamericana de Matemática Educativa 32, 600-607. México: Comité Latinoamericano de Matemática Educativa.

(2018). ¿Por qué estudiar la identidad disciplinar en la formación inicial del docente de matemáticas? Premisa, 20(77), 5-20.

Pérez-Oxté, I. y Cordero, F. (2019). Modeling and anticipation of graphical behaviors in Industrial Chemical Engineering. The role of transversality of knowledge in learning mathematics. Enviado para publicar.

Soto, D. y Cantoral, R. (2014). El discurso Matemático Escolar y la Exclusión. Una visión Socioepistemológica. Bolema- Boletim de Educação matemática, 28(50), 1525-1544. doi: 10.1590/1980-4415v28n50a25

Soto, D. y Silva-Crocci, H. (en prensa). Un programa socioepistemológico para la formación inicial del profesor de matemáticas. XXII actas Jornadas Nacionales de Educación Matemática. Santiago, Chile.

Zaldívar, D. y Briceño, E. (2019). ¿Qué podemos aprender de nuestros estudiantes? Reflexiones en torno al uso de las gráficas. Educación Matemática, 31(2), 212-240. doi: 10.24844/EM3102.09 
